\author{
Military Technical College \\ Kobry El-Kobbah, \\ Cairo, Egypt
}

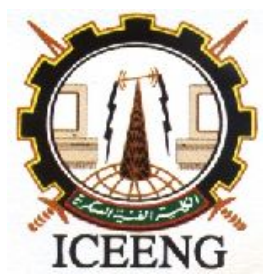

\section{$10^{\text {th }}$ International Conference on Electrical Engineering ICEENG 2016}

\title{
Patrolling Operation Using Functional Heterogeneous Two-Wheeled Mobile Robots and Viewpoints
}

\author{
By: Walaaeldin Ahmed Ghadiry*
}

\begin{abstract}
:
In this paper, the patrolling operation using two-wheeled mobile robots for monitoring a given area is considered. The two-wheeled mobile robots are considered to be functionally heterogeneous while visiting a set of viewpoints which are considered heterogeneous as well. The problem is typically related to the well-known Travelling Salesman Problem. The functionally heterogeneous two-wheeled mobile robots will only be able to visit the viewpoints that correspond to their heterogeneous functionalities. This could be related to their heterogeneous monitoring capabilities.
\end{abstract}

It is required to obtain the optimal overall minimum travel distance while preserving the constraints of the heterogeneous monitoring functionality to have the most efficient patrolling performance. A new formulation is introduced using mixed-integer programing with simulations to present the exact optimal trajectories that can represent such problems.

\section{Keywords:}

Patrolling operation, heterogeneous viewpoints, functionally heterogeneous robots, Travelling Salesman Problem 


\section{Introduction:}

Robots have been used in many and various applications that can be difficult or dangerous to be performed by a human being. The various applications for robots include both civilian and military applications, such as monitoring environmental boundaries [ HYPERLINK "" $\backslash 1$ "SSu08" 1 ], material handling 2]\}, forest fire detection [ HYPERLINK "" $\backslash 1$ "Chi15" 3 ] and patrolling operations 4]\}. In particular, the twowheeled mobile robots have the advantage of being able to turn on the spot and thus have the ability to track trajectories that are composed of straight segments [ HYPERLINK "" ll "Yan13" 5 ].

Patrolling operations are typically performed by assigning a set of monitoring locations -named the viewpoints- and a number of robots that patrol these viewpoints in a specific sequence that can affect the patrolling performance 4]\}, [ HYPERLINK "" 11 "FPa121" 6 ], 7]\}. Optimizing the overall travel distance is typically related to the well-known Travelling Salesman problem, where a salesman has to visit a number of cities, once each, before returning back to the starting point which is typically named the depot. Using a single robot is related to the single depot travelling salesman problem (mTSP), while on the other hand, having more than one robot is related to the multidepot multiple Travelling Salesmen Problem (MmTSP) [ HYPERLINK "" \l "IKa06" 8 ], 9]\} [ HYPERLINK "" \l "SYa07" 10 ] 11]\}, [ HYPERLINK "' \l "WGh15" 12 ].

The robots and viewpoints are always considered homogeneous in the literature, and the optimal trajectories required to be tracked by the robots while patrolling are driven on that assumption where any viewpoint can be possibly visited by any of the robots. Lately, very few works started to consider the possible heterogonous nature of the robots as well as the viewpoints; this makes the problem more challenging and more practical. The heterogeneous nature of the robots can be due to its physical structure or its functional performance, like having different monitoring capabilities. In this way, not every 
viewpoint can be visited by any of the robots for achieving maximum patrolling performance 13]\}.

In this paper, the problem of having heterogeneous two-wheeled mobile robots for patrolling a given area is considered. A new mixed-integer programming formulation is introduced to represent this problem with the possibility of having the number of heterogeneous viewpoints sets bigger than the number of robots, as well as having the possibility of having more than one robot starting from a single depot.

The rest of this paper is organized as follows. The problem description is introduced in Section II. Section III presents the proposed framework and the new formulation. The simulation results are provided in Section IV. Conclusions are summarized in Section V.

\section{Problem Description:}

Consider a complete undirected graph $G(V, E)$, where $V=\left\{v_{1}, v_{2}, \ldots, v_{n}\right\}$, denotes a set of $n$ viewpoints through which $m$ two-wheeled functionally heterogeneous mobile robots patrol for monitoring an area, such that $m<n$. The set $D=\left\{v_{1}, v_{2}, \ldots, v_{d}\right\}$ denotes the set of $d$ starting depots, such that $d \leq m$. Set $V^{\prime}=\left\{v_{d+1}, v_{d+2}, \ldots, v_{n}\right\}$ represents the set of $n-d$ customer viewpoints, such that $V=D \cup V^{\prime}$. Set $M=\{1, \ldots, m\}$ represents the set of $m$ wheeled mobile robots, where $m_{k}$ represents the total number of robots initially departing from depot $k$ such that $\sum_{k=1}^{d} m_{k}=m$. Set $E$ is the set of all edges connecting any two viewpoints, and is used to represent the motion trajectories to be tracked by the robots. Let $\left[c_{i j}\right]$ represent the cost matrix corresponding to the edge lengths between any two viewpoints $v_{i}$ and $v_{j}$. The distance between any two connected viewpoints is adopted as the edge weight, which implies that $c_{i j}=c_{j i}, \forall(i, j) \in E$ preserving the triangular inequality, i.e., for any three viewpoints $(i, j, k), c_{i j}+c_{j k} \geq c_{i k}$. The set $H=\left\{H_{1}, H_{2}, \ldots, H_{p}\right\} \subseteq V^{\prime}$ denotes the set of $p$ heterogeneous viewpoints subsets corresponding to the heterogeneous robots such that $p \leq m$ 
and $H_{i} \cap H_{j}=\emptyset, \forall H_{i}, H_{j} \subset H, i \neq j$. The set $L=V^{\prime} \backslash H$ is the set of common viewpoints that can be visited by any robot. Each edge is represented by a binary variable $x_{i j k l}$ which is equal 1 if the edge $(i, j)$ tracked by the robot $l$ which started from the depot $k$ is optimal and 0 otherwise.

The minimum travel distance trajectories are required to be computed such that each robot follows an appropriate trajectory connecting a subset of the viewpoints that includes its corresponding heterogeneous viewpoints subset if exists, starting from a depot and returning back to it. There are two cases of interest: (i) when a return trip is allowed, i.e., the robot can visit only one viewpoint and return to its previous viewpoint, and (ii) when a return trip is not allowed. The restriction in the second case applies to applications such as pickup and delivery, where visiting only one node is not allowed.

\section{Proposed Framework:}

For the aforementioned graph $G(V, E)$, and cost matrix $\left[c_{i j}\right]$, the patrolling operation related to the Heterogeneous Multidepot multiple Traveling Salesmen Problem can be formulated in an optimization framework as follows: Let an edge connecting any two arbitrary viewpoints $v_{i}, v_{j}$ on the trajectory of depot $k$ and tracked by a robot $l$ be represented by a binary variable $x_{i j k l}$ which will is equal 1 if the trajectory is optimal and 0 otherwise. The required cost function to be minimized can be represented by the product of each element in $\left[c_{i j}\right]$ and the corresponding variable $x_{i j k l}$, as follows:

Minimize $\sum_{l \in M} \sum_{k \in D} \sum_{j \in V \prime}\left(c_{k j} x_{k j k l}+c_{j k} x_{j k k l)}+\sum_{l \in M} \sum_{k \in D} \sum_{i \in V \prime} \sum_{j \in V} c_{i j} x_{i j k l}\right.$ 
The total trajectories departing from or arriving at any prespecified depot is equal to the initial number of robots at that depot.

$\sum_{i \in M} \sum_{j \in V,} x_{k j k l}=m_{k}$

$$
k \in D
$$

$$
\sum_{l \in M} \sum_{j \in V} x_{j k k l}=m_{k} \quad k \in D
$$

The total trajectories arriving at or departing from each customer viewpoint either from a prespecified depot or any other viewpoint is equal to one, i.e., each viewpoint is visited only once.

$$
\begin{array}{ll}
\sum_{l \in M} \sum_{k \in D} x_{k j k l}+\sum_{l \in M} \sum_{k \in D} \sum_{i \in V^{\prime}} x_{i j k l}=1 & j \in V^{\prime}, i \neq j \\
\sum_{l \in M} \sum_{k \in D} x_{j k k l}+\sum_{l \in M} \sum_{k \in D} \sum_{i \in V^{\prime}} x_{j i k l}=1 & j \in V^{\prime}, i \neq j
\end{array}
$$

The total trajectories arriving at each customer viewpoint either from a depot or from any other customer viewpoint is equal to the total trajectories departing from that customer viewpoint either to the same depot or to any other customer viewpoint. This is known by the route continuity constraint and is expressed as:

$$
x_{k j k l}+\sum_{i \in V r} x_{i j k l}-x_{j k k l}-\sum_{i \in V r} x_{j i k l}=0 \quad k \in D, l \in M, j \in V^{\prime}
$$

Each customer viewpoint can only be visited once, i.e., by only one robot and from only one starting depot.

$\sum_{k \in D} \sum_{j \in V} x_{k j k l}=1$ 
One of the most important constraints in a typical TSP is to avoid any sub-tour, which is a closed trajectory that does not include a depot from $D$ as a starting and ending point to that trajectory. Introducing two artificial variables $u_{i}$ and $u_{j}$ in the optimization framework as presented in the following inequality prevents the formation of such sub-tours. Thus, the differences between the variables corresponding to a sub-tour not including the depot will always contradict the inequality, preventing such sub-tours from being formed.

$u_{i}-u_{j}+(n-m) \Sigma_{l \in M} \sum_{k \in D} x_{i j k l} \leq n-m-1 \quad i, j \in V^{\prime}, i \neq j$

Typically in applications such as pickup and delivery, should have at least two additional nodes other than the starting depot. In this case, a formulation similar to the previous problem is used along with the following inequality:

$\sum_{l \in M} \sum_{k \in D} x_{k j k l}+\sum_{l \in M} \sum_{k \in D} x_{j k k l} \leq 1 \quad j \in V^{\prime}$

\section{Simulation Results:}

Consider the patrolling problem for a field of size $20 \mathrm{~m} \times 20 \mathrm{~m}$, where a set of $n=10$ viewpoints are to be visited. Different numbers of robots are considered with the same number of depots as well as with less number of depots. In addition, the number of heterogeneous subsets is considered to be the same as the number of robots or less than the number of robots. Using the new formulation presented in this paper, it is required to find the minimum overall distance trajectories. MATLAB was used with the Gurobi Optimizer 6.0 optimization software [ HYPERLINK "" $\backslash 1$ "Gur14" 14 ] to obtain all the results using Intel Core i7-3537U @ 2.00GHz processor with 8 GB RAM. Two scenarios are adopted for these simulations, scenario 1, where the return trip is allowed and scenario 2 , when the return trip is not allowed.

\section{Scenario 1}


Fig. 1 shows the optimal trajectories representing the minimum over all travel distance when $m=d=2, p=2, H_{1}=\left\{v_{6}\right\}, H_{2}=\left\{v_{3}, v_{4}, v_{7}\right\}$. On the other hand, Fig. 2 shows the optimal trajectories representing the minimum over all travel distance when $m=3, d=2$, with the same previous heterogeneous subsets. Figs. 3, 4 show the corresponding optimal trajectories for the same previous number of depots and robots, respectively, but with only one subset of heterogeneous viewpoints $\left(H_{1}=\left\{v_{3}, v_{4}, v_{7}\right\}\right)$.

\section{Scenario 2}

Figs. 5-8 represent the simulation results analogous to scenario 1 simulations but with the assumption that the return trip is not allowed.

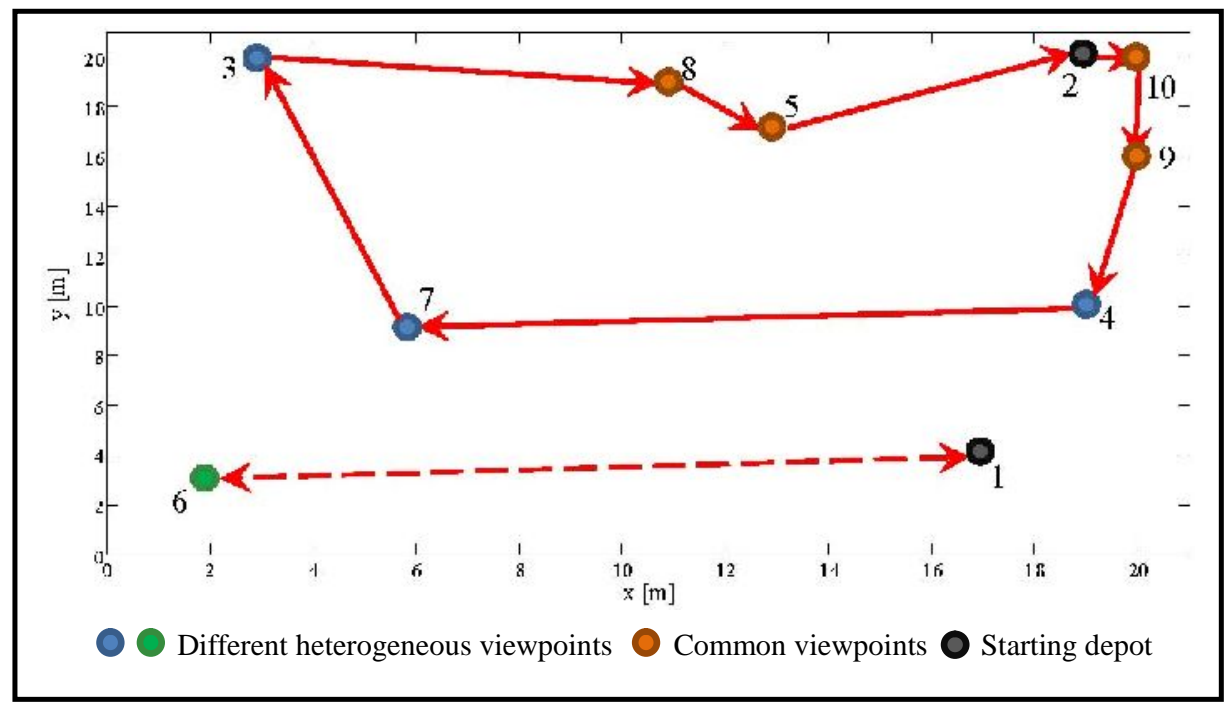

Figure (1): Optimal trajectories with two heterogeneous subsets, $H_{1}=\left\{v_{6}\right\}, H_{2}=\left\{v_{3}, v_{4}, v_{7}\right\}$ with two heterogeneous robots $m_{1}=m_{2}=1$ and $d=2$ when the return trip is allowed 


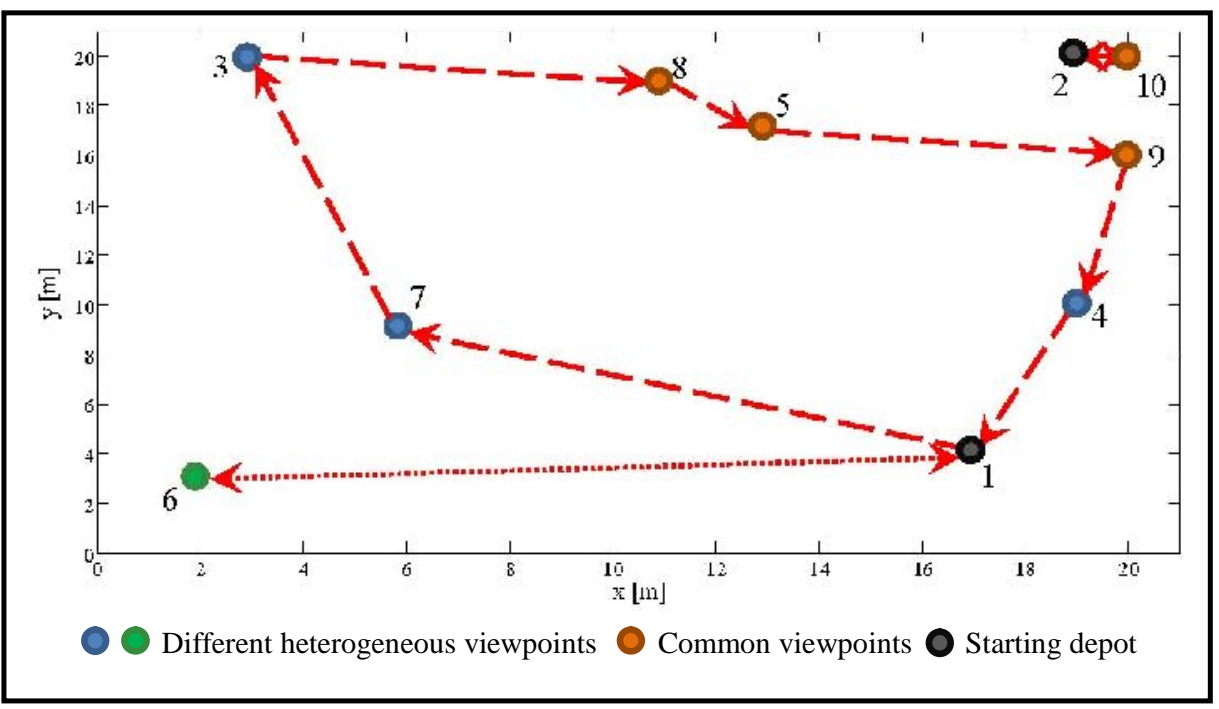

Figure (2): Optimal trajectories with two heterogeneous subsets, $H_{1}=\left\{v_{6}\right\}, H_{2}=\left\{v_{3}, v_{4}, v_{7}\right\}$ with three heterogeneous robots $m_{1}=2, m_{2}=1$ and $d=2$ when the return trip is allowed

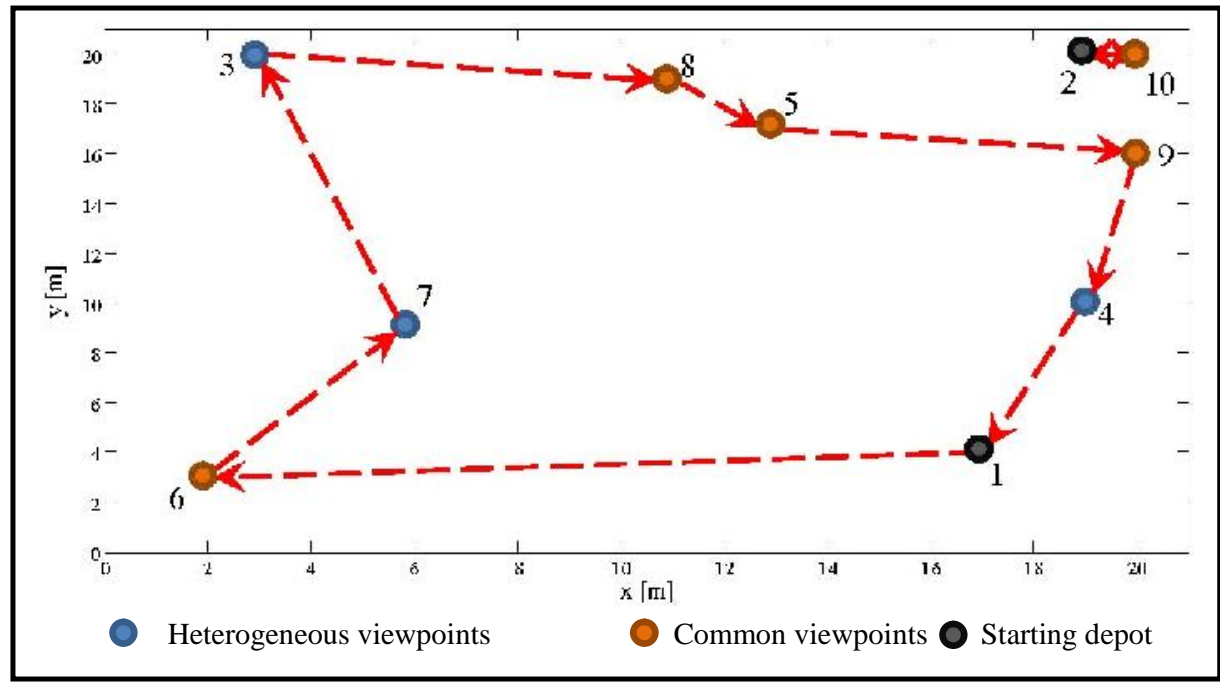

Figure (3): Optimal trajectories with one heterogeneous subset, $H_{1}=\left\{v_{3}, v_{4}, v_{7}\right\}$ two heterogeneous robots $m_{1}=m_{2}=1$ and $d=2$ when the return trip is allowed 


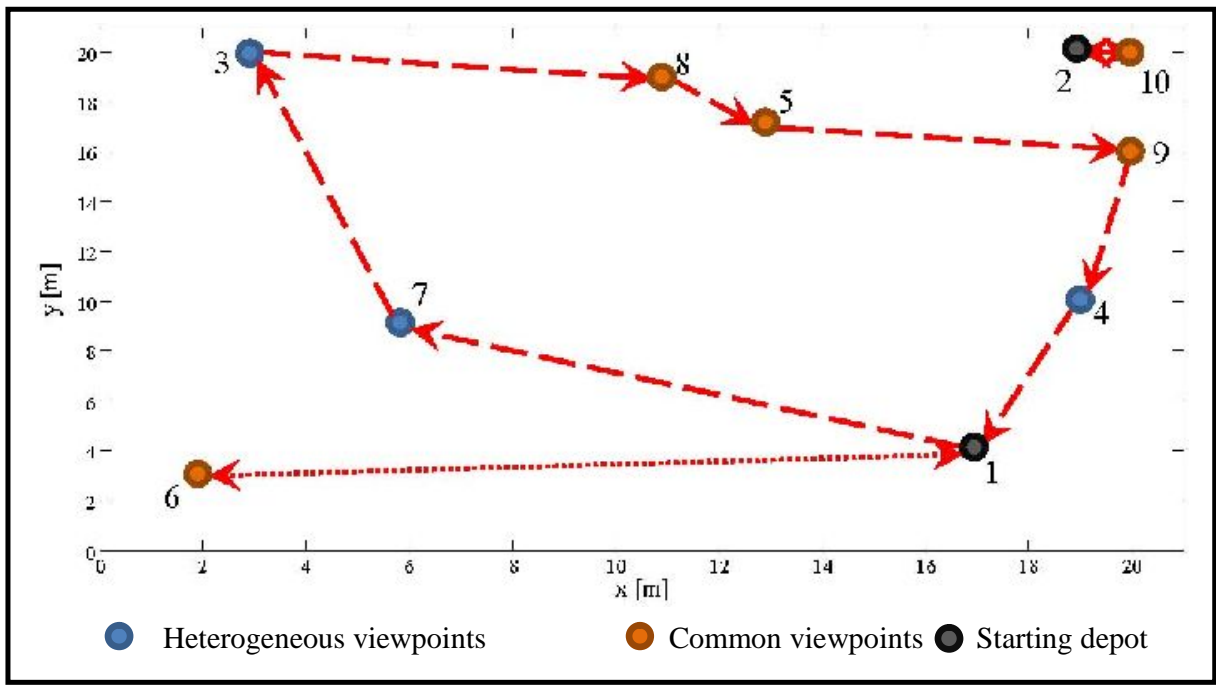

Figure (4): Optimal trajectories with one heterogeneous subset $H_{1}=\left\{v_{3}, v_{4}, v_{7}\right\}$ with three heterogeneous robots $m_{*}=2 . m_{n}=1$ and $d=2$ when the return trin is allowed

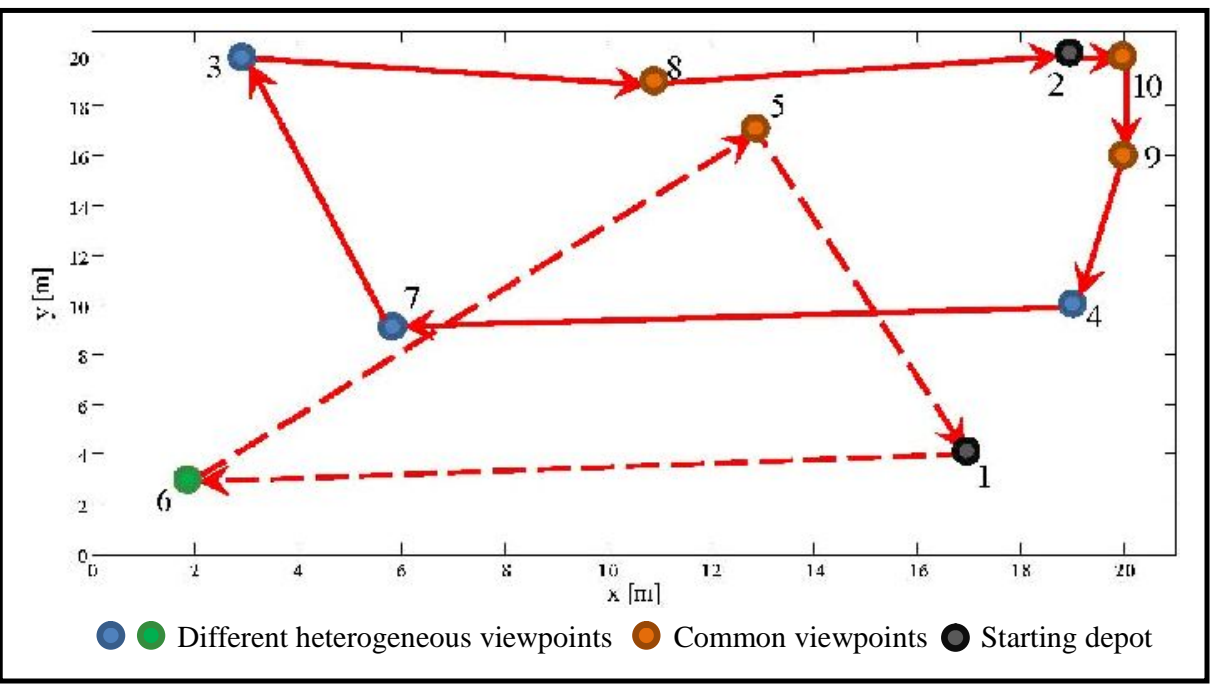

Figure (5): Optimal trajectories with two heterogeneous subsets $H_{1}=\left\{v_{6}\right\}, H_{2}=\left\{v_{3}, v_{4}, v_{7}\right\}$ with two heterogeneous robots $m_{1}=m_{2}=1$ and $d=2$ when the return trip is not allowed 


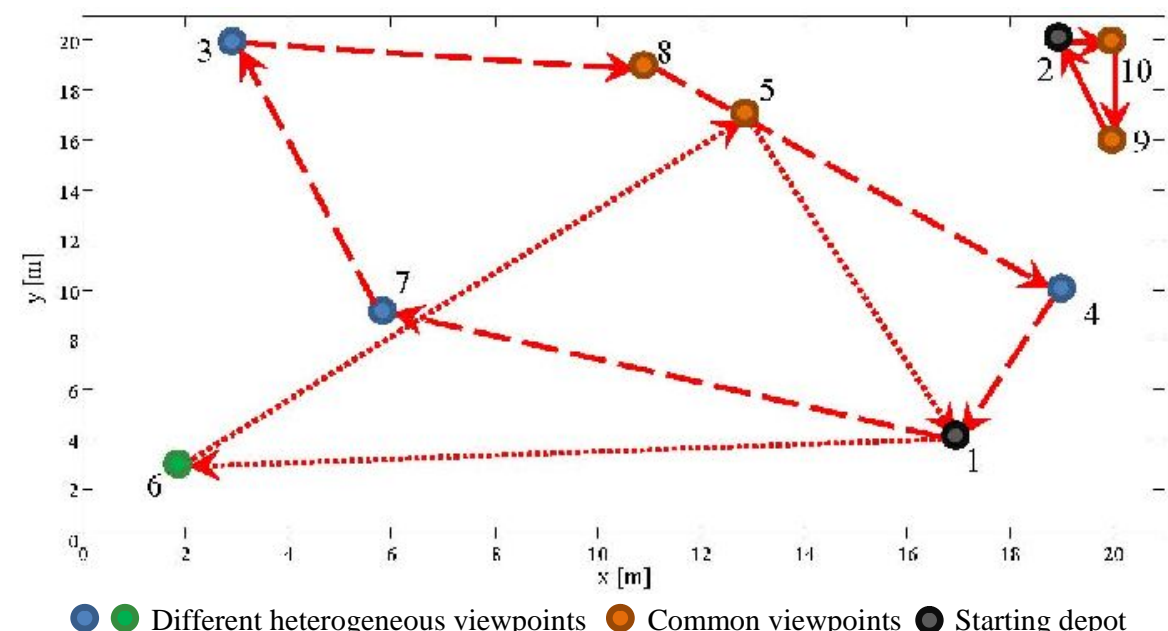

Figure (6): Optimal trajectories with two heterogeneous subsets $H_{1}=\left\{v_{6}\right\}, H_{2}=\left\{v_{3}, v_{4}, v_{7}\right\}$ with three heterogeneous robots $m_{.}=2 \mathrm{~m}-1$ and $d=2$ whon tho roturn trin is not allawod

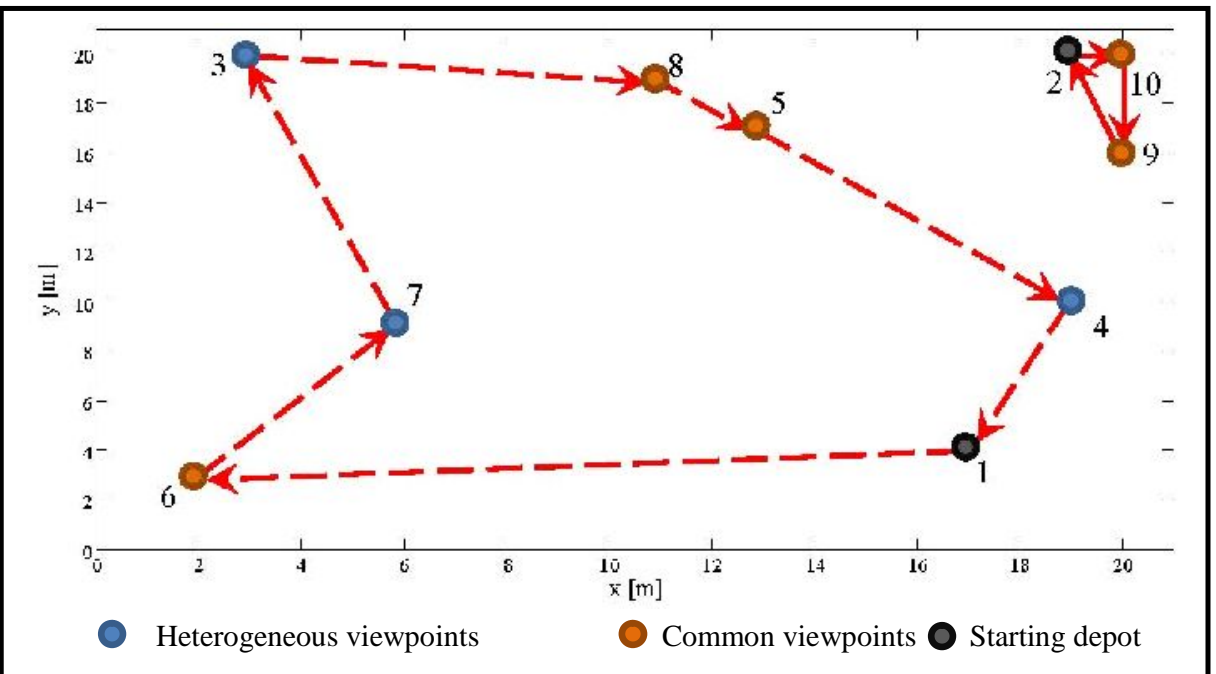

Figure (7): Optimal trajectories with one heterogeneous subset $H_{1}=\left\{v_{3}, v_{4}, v_{7}\right\}$ with two heterogeneous robots $m_{1}=m_{2}=1$ and $d=2$ when the return trip is not allowed 


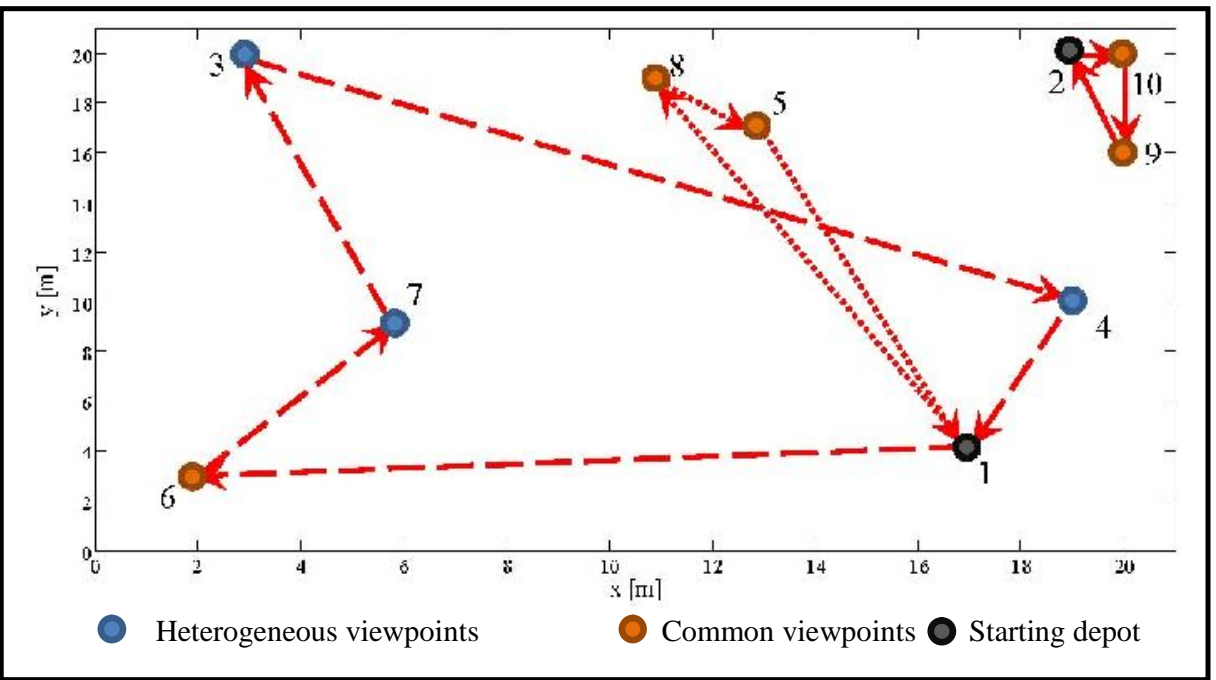

Figure (8): Optimal trajectories with one heterogeneous subset $H_{1}=\left\{v_{3}, v_{4}, v_{7}\right\}$ with three heterogeneous robots $m_{1}=2, m_{3}=1$ and $d=2$ when the return trip is not allowed

Table 1 summarizes the simulation results for the previous two scenarios with all the different cases represented in Figures 1-8.

Table 1 Overall optimal result for Scenarios 1 and 2

\begin{tabular}{|c|c|c|c|c|c|c|c|c|}
\hline & \multicolumn{4}{|c|}{$\begin{array}{c}\text { Scenario 1 } \\
\text { Return trip is allowed }\end{array}$} & \multicolumn{4}{|c|}{$\begin{array}{c}\text { Scenario } 2 \\
\text { Return trip is not allowed }\end{array}$} \\
\hline $\begin{array}{c}\text { Number of starting } \\
\text { depots }\end{array}$ & \multicolumn{8}{|c|}{$d=2$} \\
\hline Number of robots & $m=2$ & $m=3$ & $m=2$ & $m=3$ & $m=2$ & $m=3$ & $m=2$ & $m=3$ \\
\hline $\begin{array}{l}\text { Initial number of } \\
\text { robots at each depot }\end{array}$ & $\begin{array}{l}m_{1}=1 \\
m_{2}=1\end{array}$ & $\begin{array}{l}m_{1}=1 \\
m_{2}=2\end{array}$ & $\begin{array}{l}m_{1}=1 \\
m_{2}=1\end{array}$ & $\begin{array}{l}m_{1}=1 \\
m_{2}=2\end{array}$ & $\begin{array}{l}m_{1}=1 \\
m_{2}=1\end{array}$ & $\begin{array}{l}m_{1}=1 \\
m_{2}=2\end{array}$ & $\begin{array}{l}m_{1}=1 \\
m_{2}=1\end{array}$ & $\begin{array}{l}m_{1}=1 \\
m_{2}=2\end{array}$ \\
\hline $\begin{array}{c}\text { Number of } \\
\text { heterogeneous } \\
\text { subsets }\end{array}$ & \multicolumn{2}{|c|}{$p=2$} & \multicolumn{2}{|c|}{$p=1$} & \multicolumn{2}{|c|}{$p=2$} & \multicolumn{2}{|c|}{$p=1$} \\
\hline $\begin{array}{l}\text { Heterogeneous } \\
\text { subsets }\end{array}$ & \multicolumn{2}{|c|}{$\begin{array}{c}H_{1}=\left\{v_{6}\right\} \\
H_{2}=\left\{v_{3}, v_{4}, v_{7}\right\}\end{array}$} & \multicolumn{2}{|c|}{$H_{1}=\left\{v_{3}, v_{4}, v_{7}\right\}$} & \multicolumn{2}{|c|}{$\begin{array}{c}H_{1}=\left\{v_{6}\right\} \\
H_{2}=\left\{v_{3}, v_{4}, v_{7}\right\}\end{array}$} & \multicolumn{2}{|c|}{$H_{1}=\left\{v_{3}, v_{4}, v_{7}\right]$} \\
\hline Total travel distance & $83.1884 \mathrm{~m}$ & $85.9205 \mathrm{~m}$ & $66.0152 \mathrm{~m}$ & $85.9205 \mathrm{~m}$ & $98.0867 \mathrm{~m}$ & $105.4756 \mathrm{~m}$ & $69.2040 \mathrm{~m}$ & $100.5472 \mathrm{~m}$ \\
\hline Computation time & $0.08 \mathrm{~s}$ & $0.07 \mathrm{~s}$ & $0.03 \mathrm{~s}$ & $0.1 \mathrm{~s}$ & $0.125 \mathrm{~s}$ & $0.04 \mathrm{~s}$ & $0.03 \mathrm{~s}$ & $0.09 \mathrm{~s}$ \\
\hline
\end{tabular}




\section{Conclusions:}

This paper presents a new mixed-integer programming formulation for patrolling operations based on heterogeneous travelling salesmen problem. The simulations done using this formulation resulted in the optimal minimum-distance trajectories for patrolling a given area while preserving the constraints of visiting the heterogeneous subsets of the viewpoints with functionally heterogeneous robots. Two scenarios are tackled, when the return trip is allowed and when it is not allowed. In addition, two special cases are introduced among the simulations results, the first is when the number of robots is more than the starting depots and the second is when the number of heterogeneous subsets is less than the number of heterogeneous robots.

\section{References:}

[1] S. Susca, S. Martinez, and F. Bullo, "Monitoring environmental boundaries with a robotic sensor network," IEEE Trans. Control Syst. Technol., vol. 16, no. 2, pp. 288-296, 2008.

[2] S. Read, A. Van der Merwe, S. Matope, A. Smit, and M. Mueller, "An intuitive teachable micro material handling robot with Van der Waals gripper design and development," in Robotics and Mechatronics Conference of South Africa (ROBOMECH), 2012 5th, Gauteng, 2012, pp. 1-6.

[3] Chi Yuan, Zhixiang Liu, and Youmin Zhang, "UAV-based forest fire detection and tracking using image processing techniques," in International Conference on Unmanned Aircraft Systems (ICUAS), Denver, CO, 2015, pp. 639 - 643.

[4] F. Pasqualett, A. Franch, and F. Bullo, "On cooperative patrolling: optimal trajectories, complexity analysis, and approximation algorithms," IEEE Trans. Robot, vol. 28, no. 3, pp. 592-606, 2012.

[5] BoYang, N. Hanajima, A. Yamamoto, M. Ayamura, and Jun Dai, "Path-generating regulator along a straight passage for two-wheeled mobile robots," in International Conference on Intelligent Robots and Systems (IROS), Tokyo, 2013, pp. 4837 - 4844. 
[6] F. Pasqualetti, J. W. Durham, and F. Bullo, "Cooperative patrolling via weighted tours: performance analysis and distributed Algorithms," IEEE Trans. on Robotics, vol. 16, pp. 11811188, 2012.

[7] D. Portugal and R. P. Rocha, "Distributed multi-robot patrol: A scalable and fault-tolerant framework," Robotics and Autonomous Systems, vol. 61, pp. 1572-1587, 2013.

[8] I. Kara and T. Bektas, "Integer linear programming formulations of multiple salesman problems and its variations," European Journal of Operational Research, pp. 1449-1458, 2006.

[9] Togla Bektas, "The multiple traveling salesman problem: an overview of formulations and solution procedures," Omega, vol. 34, no. 3, pp. 209-219, 2006.

[10] S. Yadlapalli, W. A. Malik, S. Darbha, and M. Pachter, "A Lagrangian-based algorithm for a multiple depot, multiple traveling salesmen problem," in ACC, New York City, 2007.

[11] W. Ghadiry, J. Habibi, A. G. Aghdam, and Y. M. Zhang, "A new formulation for energyefficient trajectory optimization in patrolling problem with non-pre-defined starting depot," in 10th International Conference on Intelligent Unmanned Systems (ICIUS, 2014), Montreal, Qc, 2014.

[12] W. Ghadiry, J. Habibi, A. G. Aghdam, and Y. M. Zhang, "Generalized formulation for trajectory optimization in patrolling problems," in 28th annual IEEE Canadian Conference on Electrical and Computer Engineering, Halifax, 2015, pp. 231-236.

[13] Kaarthik Sundary and Sivakumar Rathinam, "An exact algorithm for a heterogeneous, multiple depot, multiple travelling salesman problem," in 2015 International Conference on Unmanned Aircraft Systems (ICUAS), Denver, Colorado, 2015, pp. 366-371.

[14] Gurobi_6.0, Optimization software, 2014. 